\title{
THE DIAMOND PROJECT: AN ADVANCED LIGHT SOURCE FOR THE UK
}

\author{
J A Clarke, D M Dykes, M T Heron, D J Holder, J Kay, N Marks, C Nave, M W Poole, R J Reid, \\ R Ryder, S L Smith, V P Suller, CLRC Daresbury Laboratory, UK \\ R J Mason, CLRC Rutherford Appleton Laboratory, Didcot, UK
}

\begin{abstract}
The DIAMOND project was formally approved in 1998 and in March 2000 a site immediately adjacent to the Rutherford Appleton Laboratory near Oxford was selected. Funding of the project has been offered by three partners: the UK and French governments and the Wellcome Trust, the world's largest medical research charity. The formal Design Phase was initiated in November 2000 with scheduled completion early in 2002; a storage ring with an energy of $3 \mathrm{GeV}$, a 24 cell structure and a circumference well over $500 \mathrm{~m}$ has already been confirmed, together with other key parameters. Intensive lattice optimisation is under way and is described in more detail in an accompanying paper. In the present one we will give a project overview and explain key design decisions and overall progress, including the facility specification which includes a superconducting RF system. Planned beam commissioning will be in 2006 .
\end{abstract}

\section{BACKGROUND}

The DIAMOND facility is intended to provide the UK with a very powerful source of $\mathrm{x}$-rays to replace the existing national source (SRS) at Daresbury. It forms the centre piece of a 'three ring strategy' that has been proposed to cover the full range from low to very high photon outputs [1]. The initial concept of a racetrack storage ring of modest beam brightness [2] subsequently evolved into a larger, very high brightness version [3] that was further expanded after the French government joined the project in 1999 [4]. This followed an earlier joint project funding announcement in 1998 by the UK government and the Wellcome Trust, an endowed research charity.

In March 2000, after much debate, the three partners announced their decision to locate the new facility on land adjacent to the Rutherford Appleton Laboratory (RAL) near Oxford. This is effectively a green field site, although the RAL infrastructure (probably upgraded) was seen as an important addition to the DIAMOND facilities.

The initial scientific case had been established by 1994 but more recently the growing use of synchrotron radiation for genomics and proteonics has led to a perceived increase in the need for high brightness radiation above the few $\mathrm{keV}$ region for which DIAMOND was originally targeted; in particular, at least the $12.7 \mathrm{keV}$ selenium edge must be reachable. This led to a reconsideration of all of the basic DIAMOND parameters in order to match it to this specification, as will be discussed below.

In November 2000, after a period of further user consultation and of agreeing some of these critical parameters, a formal Design Phase was initiated and is expected to be completed early in 2002. Design work is largely being undertaken by Daresbury staff under a contract with the Office of Science and Technology, the UK government agency with specific responsibility for DIAMOND.

\section{KEY DECISIONS}

Before commencing the detailed Design Phase it was agreed that decisions should be made on a number of critical parameters, relating these to the performance demands of the user community.

\subsection{Energy}

Selection of the storage ring energy is based on a wide variety of criteria. The principal technical decision concerns the delivery of radiation with adequate properties. At $3 \mathrm{GeV}$ the undulators are highly optimised in the medium energy region from $500 \mathrm{eV}$ to $5 \mathrm{keV}$, but less so above $10 \mathrm{keV}$. A detailed assessment has been carried out of the consequences of an energy increase to $3.5 \mathrm{GeV}$ and it is clear that there is a significant project cost penalty, not only to the storage ring systems but also to the full energy injector booster synchrotron. Delivery of high brightness photons at the important $12.7 \mathrm{keV}$ energy can however be ensured at the lower $3 \mathrm{GeV}$ level by use of high performance undulators, in particular exploiting higher harmonic output. There will also be a need to operate such undulators at low gaps; for the purposes of the decision a minimum gap of $10 \mathrm{~mm}$ has been assumed, although in that case the undulator length has been restricted to no more than $2 \mathrm{~m}$. For this example the brightness at $3 \mathrm{GeV}$ reaches $5 \times 10^{19} \mathrm{sec}^{-1} \mathrm{~mm}^{-2} \mathrm{mrad}^{-2}$, which is within a factor 10 of the $6 \mathrm{GeV}$ ESRF ring.

DIAMOND is also intended to be a very high flux facility and this is ensured by a range of possible multipole wigglers (MPW) that generate output up to and beyond $100 \mathrm{keV}$.

Figure 1 illustrates the anticipated spectral output from the present DIAMOND design and also includes some more speculative insertion devices (IDs) such as an invacuo undulator, a $3.5 \mathrm{~T}$ MPW and a $10 \mathrm{~T}$ wavelength 
shifter. Such IDs provide an upgrade path for the project after initial operations are established. As a result of these studies the $3 \mathrm{GeV}$ energy has now been confirmed.

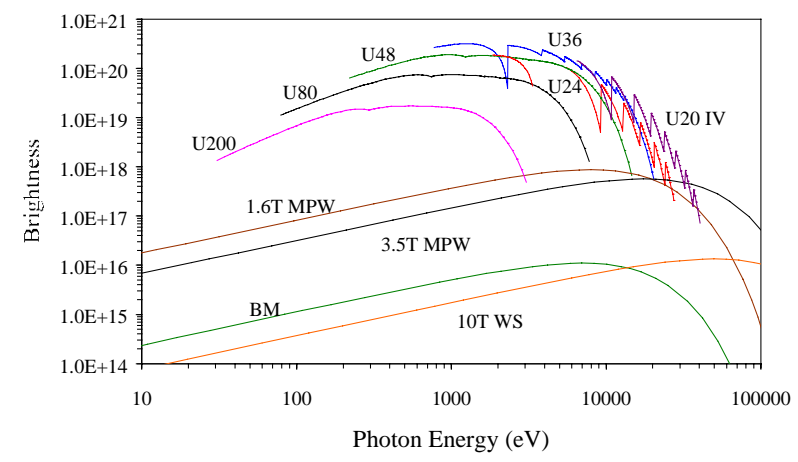

Fig 1 Spectral output from DIAMOND

\subsection{Lattice Layout}

Much early DIAMOND design work was carried out on a 16 cell DBA lattice and a beam dynamics design procedure involving 4-cell units was developed. On increasing the ring size this unit approach was preserved and a 24 cell solution has now been selected. This is also compatible with the inclusion of six longer straight sections in a symmetric arrangement. Two of these will be utilised for injection and the RF system and a length of $8 \mathrm{~m}$ has been selected as adequate for this purpose; the remaining four straights are then available for user IDs.

The standard straight length has recently been increased slightly to allow IDs up to $5 \mathrm{~m}$ to be installed; it also increases flexibility for multiple IDs in a single straight as an option. The resultant straight lengths are compared in figure 2 with those of other $3^{\text {rd }}$ generation light sources.

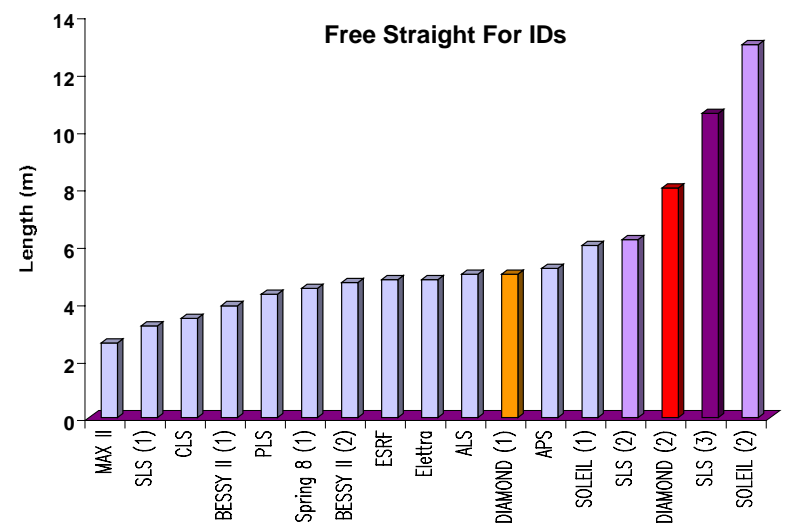

Fig 2 Straight length comparisons

The translation of this physics lattice into an engineering design is discussed below.

\subsection{Beam Current and Lifetime}

Extensive consultation with the UK user community has led to a minimum lifetime specification of 10 hours being adopted, with a preferred target of double this. In practice the DIAMOND stored beam suffers similar loss rates from both gas and Touschek scattering processes [5] and the higher lifetime target will be difficult to achieve. A minimum (full) vertical vacuum aperture of $7 \mathrm{~mm}$ has been assumed for an ID straight in these loss calculations, leading to the $10 \mathrm{~mm}$ minimum gap undulator assumption of the spectral output results.

A beam current of $300 \mathrm{~mA}$ has been selected and is used for all present performance calculations. This produces $300 \mathrm{~kW}$ radiation emission from the ring bending magnets and up to $200 \mathrm{~kW}$ more from the IDs (depending on the number of high field devices eventually installed). However consideration is being given to ensuring that technical systems are rated to 500 $\mathrm{mA}$ to permit future upgrades.

\section{DESIGN STUDIES}

This section discusses progress made in the first six months of the formal Design Phase. However until the latter is completed it cannot yet be considered to be a finalised design solution.

\subsection{Storage Ring Dynamics}

Extensive lattice studies have been continuing and the latest results are reported in an accompanying paper [5]. Optimisations have produced a high performance with an emittance as low as $2 \mathrm{~nm}$-rads in the non-zero dispersion mode. Careful control of phase advances has been employed and the resultant matching has achieved the target lattice parameters, including a suitable mix of beta values. Non-linear optimisation is still under way but promising results have already been achieved using four harmonic sextupole families in addition to the two chromatic ones: on-momentum dynamic (half) aperture exceeds $20 \mathrm{~mm} \times 10 \mathrm{~mm}(\mathrm{H} \mathrm{x} \mathrm{V})$ and good progress towards the desired $4 \%$ momentum aperture can also be reported. Such a good momentum aperture will be an important contribution towards meeting the stored beam lifetime target, and is typical of the challenges set to the latest advanced light source designs.

Establishing this lattice has also involved careful assessment of component locations, both from an accelerator physics and engineering perspective. This iterative design interaction has now produced a realistic layout with a circumference of about $560 \mathrm{~m}$ and a corresponding escalation of previous size of building.

In order to optimise beam lifetime in high performance storage rings there has been much interest in top-up injection schemes; the most experience so far is at the APS facility which has demonstrated routine achievement of $0.3 \%$ current stability over many hours. An international Workshop organised by the DIAMOND project in March 2001 reviewed design schemes and operational experience, together with implications for new projects and their user communities. The positive 
conclusions have encouraged the incorporation of top-up into the DIAMOND design and this is now to be actively pursued. It would certainly allow reduced gap IDs to be incorporated into the lattice, although this is considered to be a later upgrade step after initial operations are established. Serious issues that have still to be addressed include the necessity of a very high efficiency injection process; a full beam loss simulation and conformity with regulatory radiation restrictions; user tolerance and potential gating during transient perturbations; and the implications for injector equipment.

\subsection{Technical Issues}

A variety of technical areas have been progressed during the Design Phase. There has also been close interaction with user representatives and formal Advisory Committees. One topic not yet fully resolved is the extent of utilisation of DIAMOND for low energy radiation. Although there is still some prospect that a dedicated low energy source [6] might eventually be constructed in the UK there is already considerable demand for access to DIAMOND in at least two important areas. Up to 20 ports on DIAMOND bending magnets will be available and some could provide infrared output if the absorber within the vacuum chamber was to be modified to permit the large vertical opening angle (ca $30 \mathrm{mrad}$ ) to be transmitted onto a close-in slotted mirror. Circular dichroism (CD) is another important low energy (UV/visible) topic whose inclusion on DIAMOND is being assessed. In this case the required angular aperture is much smaller $(8 \mathrm{mrad})$ but the forward transmission interferes with some downstream storage ring magnets. Engineering solutions for both these applications appear possible.

The RF system for DIAMOND is a large one, necessitating about 4-5 MV for adequate momentum acceptance of $4 \%$ and beam power replacement of at least $500 \mathrm{~kW}$. The original concept of a normal conducting system would need 5 or 6 cavities and might not fit in a single straight section. Furthermore the coupled bunch instabilities driven by the cavity HOMs would be severe and necessitate comprehensive, expensive countermeasures. Another DIAMOND sponsored international Workshop convinced the design team that a superconducting (SRF) solution was more attractive: capital costs would be similar but running costs lower; feedback systems simpler with reduced beam instability problems; and reliability could be ensured by conservative operating ratings and use of a proven design solution. A decision has therefore been taken to adopt the successful Cornell CESR cavity design, modified slightly to incorporate 3 separate cavities in a single $8 \mathrm{~m}$ DIAMOND straight. The project team is also collaborating with Cornell and a potential manufacturer to raise the power input to these cavities by improvements to the input coupler system.
Significant design progress has been made on the storage ring magnet systems. Relatively low current densities of 3-4 $\mathrm{A} / \mathrm{mm}^{-2}$ will reduce the thermal loading effects and improve component stabilities. Attention has been given to a careful assessment of magnet lengths and coil overhangs in order to establish the ring layout. Magnet apertures have yet to be finalised, based on the beam dynamics specifications, but a smooth aperture through the achromats is the preferred solution. Consideration is also being given to omission of in-situ bakeout, with consequent significant savings on apertures and costs; few modern electron rings employ such bakeout, relying instead on effective beam cleaning.

A feature of the DIAMOND design will be the quest for operational flexibility. If the lattice can demonstrate this (eg variable betas between cells) then it is intended that all quadrupoles and sextupoles will have individual power supplies.

The $3 \mathrm{GeV}$ booster synchrotron is itself a significant design challenge and cost optimisation plus operational efficiency must be matched. Furthermore adoption of topup to the storage ring places additional demands on the booster specification. The selected solution is a 2 -fold symmetric missing magnet FODO lattice that delivers an extracted beam emittance of about $125 \mathrm{~nm}$-rads from a ring about $150 \mathrm{~m}$ circumference. It will have 36 dipoles and 8 straights each providing over $3 \mathrm{~m}$ for equipment. An outstanding major issue is the main dipole power supply: a conventional White circuit is not well suited to intermittent top-up mode and alternatives are being pursued.

A final technical topic is the site selection and associated building layout and specification. Vibration analysis of the presently preferred location on the East side of the RAL site has given acceptable results but long term ground settlement projections have forced the adoption of a multiple piling solution for building foundations. The large ring circumference has also led to an annular building design some $235 \mathrm{~m}$ in diameter, providing beam lines $40 \mathrm{~m}$ in length external to the shielding.

\section{REFERENCES}

[1] V P Suller, "A source design strategy providing $5 \mathrm{eV}$ 100keV photons", Journ Synch Rad 1 (1994) 5

[2] M W Poole \& V P Suller, "Design progress on DIAMOND, a proposed UK national light source", EPAC'96, Sitges

[3] A A Chesworth et al, "DIAMOND: a UK national light source project", PAC'99, New York

[4] M W Poole et al, "Evolution of the DIAMOND light source", EPAC'00, Vienna

[5] J A Clarke et al, "Recent developments in the DIAMOND storage ring design", these Proceedings

[6] J A Clarke et al, "Prospects for a $4^{\text {th }}$ generation light source for the UK", these Proceedings 